\title{
Influence of training protocols on text input speed on a computer in individuals with cervical spinal cord injury: a randomised controlled trial
}

\author{
Samuel Pouplin $1,2,3,4 \cdot$ Djamel Bensmail $^{1,2,3,4} \cdot$ Isabelle Vaugier $^{4} \cdot$ Axelle Gelineau $^{1,2,3} \cdot$ Sandra Pottier $^{4}$. \\ Nicolas Roche ${ }^{3,4,5}$
}

Received: 3 August 2018 / Revised: 13 February 2019 / Accepted: 13 February 2019 / Published online: 27 February 2019

(c) International Spinal Cord Society 2019

\begin{abstract}
Study design Randomised controlled trial.

Objectives To evaluate the impact of two different word prediction software (WPS) training protocols on text input speed (TIS) in people with tetraplegia and to determine which was the most effective.

Setting Rehabilitation department, Garches, France.

Methods Participants with neurological levels between C6 and C8 were allocated to one of three different groups. The REHAB group underwent training with an occupational therapist. The SELF group carried out a standardised home selftraining with a written training guide. The CONTROL group had no training. Participants were assessed at day 15 (D15) and day 30 (D30). The primary outcome was a copying task with and without WPS (WITH and WITHOUT).

Results Forty-two participants (mean age \pm SD of $39.8 \pm 12$ ) were included and 38 completed the study. At D30, the mean (95\% confidence interval) difference in TIS between the CONTROL and SELF groups was 3.8 [ -1.7 to 9.4] characters per minute (cpm) $(p=0.23)$, between the REHAB and SELF groups was 12.9 [7.4 to 18.4] cpm $(p<0.001)$, and between the REHAB and CONTROL groups was $9.1[3.5$ to 14.6$] \mathrm{cpm}(p<0.001)$.

Conclusions The results of this study showed that occupational therapist-supervised training improved TIS but word prediction software did not increase TIS. These results suggest that supervised training should be provided to all individuals who are prescribed with devices and systems to facilitate computer access in order to increase their TIS.
\end{abstract}

Supplementary information The online version of this article (https:// doi.org/10.1038/s41393-019-0265-5) contains supplementary material, which is available to authorised users.

Samuel Pouplin

samuel.pouplin@aphp.fr

pouplinsamuel@gmail.com

1 New Technologies Plate-Form, AP-HP, Raymond Poincaré Teaching Hospital, Garches, France

2 Physical Medicine and Rehabilitation Department, AP-HP, Raymond Poincaré Teaching Hospital, Garches, France

3 Inserm Unit 1179, Team 3: Technologies and Innovative Therapies Applied to Neuromuscular Diseases, University of Versailles, St-Quentin-en-Yvelines, France

4 Clinical Innovations Centre 1429, AP-HP, Raymond Poincaré Teaching Hospital, Garches, France

5 Physiology-Functional Testing Ward, AP-HP, Raymond Poincaré Teaching Hospital, Garches, France

\section{Introduction}

In France, the incidence of spinal cord injury (SCI) is approximately 19.4 persons per million per year, which is around 930 new cases per year [1]. Injuries resulting in tetraplegia account for almost half of traumatic SCIs (38$56 \%$ ) [2]. Computers and internet access play an important role in facilitating participation in society for people with tetraplegia [3,4] and a variety of computer access devices have been developed to compensate for sensorimotor impairments, depending on the level of the lesion [5-8]. Despite the development of methods to increase text input speed (TIS), such as speech recognition systems [9-11] and word prediction software (WPS) [9, 12], TIS remains lower for people with tetraplegia compared with people without disabilities $[9,10]$. Increasing TIS can be very important in certain situations, such as in school or the workplace, where efficiency is required. Twenty to $47 \%$ of people with SCI return to professional activities [13] although there are 
currently no data available regarding the number of people with tetraplegia who return to work. WPS is recommended to increase TIS in situations where speech recognition software cannot be used, for example, in the classroom or an open plan office. In addition, some people prefer to maintain their privacy when inputting text.

The effects of WPS on TIS are unclear in the literature [9]; some studies in individuals with SCI found that WPS increased TIS, while others found that it had no effect $[9,14]$. These differences could be attributed to differences in the WPS settings used in each study $[15,16]$ and the fact that time spent searching for words in the prediction list may increase cognitive load. The results of studies do appear to suggest that WPS does not increase TIS in individuals with C6-C8 SCI $[15,16]$. However, the work by our team suggests that training in the use of WPS can increase TIS. In recent study, we showed that the disparity of currently published results could be due to a lack of specific training (WPS-oriented personalised training specifically aimed at individuals with tetraplegia) [17].

To date, only one study has evaluated the effect of a WPS training protocol on TIS in people with SCI. The study, published in the mid-1990s, included six individuals with tetraplegia and eight non-disabled control participants and found an $18 \%$ decrease in key selection time at the end of seven training sessions in both groups $[14,18]$. Another study in five participants without disabilities found TIS improved following 20 45-min training sessions in keyboard use over a period of 4 weeks [19]. Similarly, Ward et al. showed improvements in TIS following six daily halfhour training sessions in 10 individuals without disabilities using a dynamic virtual keyboard and a standard mouse [20]. All of these studies were performed either in university laboratories or rehabilitation departments.

Only two studies evaluated self-training at home. One included 10 individuals with sensorimotor impairment who underwent training sessions on a speech synthesiser equipped with WPS, for $1 \mathrm{~h}$ each day for 10 days. They were supervised by a health professional, initially in a healthcare setting, before continuing at home for a further 2 months. The results were disparate, ranging from a $15 \%$ decrease in TIS using WPS to a $34 \%$ increase [21]. The other study was carried out by our group and evaluated a TIS self-training protocol using either a dynamic on-screen keyboard or an on-screen AZERTY keyboard (the French version of the English keyboard), both equipped with WPS. Ten people with sensorimotor impairment borrowed the devices for 2 months and were asked to train themselves (without additional support) to use it at home; TIS did not improve in any of the participants [22].

None of these studies evaluated the effect of different training protocols and generally lacked robust methodology. The samples often included individuals with impairments of different aetiology, were generally small and lacked power to enable statistical analysis of the impact of WPS on TIS or the effect of WPS training on WPS usage.

We therefore designed this study to evaluate the impact of different WPS training protocols on TIS in people with tetraplegia and to determine which was the most effective. This was based on our working hypothesis that the presence of a therapist to explain all the possibilities provided by WPS would improve the use of WPS and thus TIS.

We compared three different groups: two training groups and one control group. The REHAB group carried out a training protocol in a Physical Medicine and Rehabilitation department that was supervised by an occupational therapist over a period of 4 weeks ( $45 \mathrm{~min}$ each day three times per week). The SELF group carried out a self-training protocol at home that was standardised but unsupervised. The CONTROL group received no specific WPS training.

We hypothesised that the supervised protocol would lead to the greatest improvements in TIS, based on data in the literature [14-19]. We considered a difference of two words per minute between groups to be clinically meaningful, based on a previous study by our group [15].

\section{Methods}

\section{Participants}

Between September 2014 and March 2017, participants with tetraplegia who were outpatients in our University teaching hospital were invited to participate in this study by a single occupational therapist. Probability sampling was used. Inclusion criteria were: (i) aged over 18 years; (ii) sustained an AIS A or B SCI with a neurological level between $\mathrm{C} 6$ and $\mathrm{C} 8$ within the preceding 6 months; (iii) already a computer user prior to the SCI (with a standard keyboard); and (iv) did not use WPS prior to the SCI. There was a single non-inclusion criterion: visual impairment preventing computer use.

The study was approved by the local ethics committee and all participants provided written informed consent before participation. Data collection was finalised in April 2017. The study was registered prospectively on ClinicalTrials.gov (NCT01953666).

\section{Materials}

For the evaluation, a Dell XPS computer equipped with Skippy WPS was used. This WPS functions by suggesting words that begin with the first letter typed. The words are then refined as more letters are typed. The user can then simply select the word without having to type each letter, thus reducing the number of keystrokes required and 
theoretically increasing TIS. This WPS was chosen because previous work showed that Skippy software was the most used by professionals and individuals with tetraplegia in France [12]. For the training, the participants used their own home computer and we provided Skippy WPS. The WPS was configured with six words displayed in a horizontal word list across the top of the screen $[16,23]$. The parameters 'automatic learning of new words' and 'frequency of use' were activated [15] during the training and the evaluation. All participants accessed the computer using a standard keyboard and either pressed keys using the metacarpophalangeal joints of their fifth digits bilaterally with the forearms supinated, or used typing splints.

\section{Study design}

The study design was a randomised controlled trial. The between participants factor was training (REHAB, SELF, and CONTROL) (three factors), and the within participants factors were word prediction (WITH and WITHOUT) (two factors) and time (day 0 (D0), day 15 (D15), and day 30 (D30)) (three factors) reflecting a $3 \times 2 \times 3$ design. Sex, age, level of education, duration of computer usage (years), and the frequency of computer and word processing usage ( $>$ three times per week; $\leqslant$ three times and $>$ once time per week; <once per week) were recorded by means of a questionnaire. Each participant was assigned to one of the three groups according to a randomisation list generated by a computer and delivered by means of sealed envelopes. Randomisation was balanced 1:1:1 between the three groups for blocks of size three, six, or nine participants in a random sequence. The randomisation list was checked by an independent statistician (details of the training protocols are provided in the appendix). The three groups were as follows:

1. Supervised training group (REHAB): participants in this group underwent training with an occupational therapist (not the author) for $45 \mathrm{~min}$, three times a week for a period of 4 weeks. The standardised training involved copying text (from a daily newspaper) and free-text input on a subject topic of the participant's choice (e.g., writing an email, describing their day etc.), both with and without WPS. The occupational therapist sat beside the participant throughout the training and indicated verbally when the WPS provided the correct word.

2. Home self-training group (SELF): the participants in this group followed a standardised protocol designed by an occupational therapist for $15 \mathrm{~min}$ per day, five times a week for 4 weeks. They were provided with training documentation. This duration of self-training was chosen based on data from an unpublished survey of participants with tetraplegia that showed $15 \mathrm{~min}$ per day was the maximum amount of training that could be performed at home. The training document included text-copying and free-text input exercises, both with and without WPS.

3. No training group (CONTROL): this group received no specific training.

Both the supervised (REHAB) and home self-training (SELF) protocols were developed using findings in the literature $[14,18,19]$, as well as our own clinical experience. Unfortunately, there is currently no evidence base for the type of training that could increase TIS. All three groups underwent the same evaluation on D0, D15, and D30. The occupational therapist (the author) who assessed the outcomes was blinded to group assignment. On D0, the WPS was explained to each participant and then they completed a 5-min training session using WPS for a text-copying task. The evaluation then consisted of two 10-min copying tasks in a randomised order:

A. Copying task without WPS (WITHOUT condition).

B. Copying task with WPS (WITH condition).

A 5-min break was allowed after each copying task. Two 600-word texts of similar complexity (level of education of 6 years, i.e., age 12 reading level) were used from a speech and language therapy book [24] with an average \pm standard deviations (SD) word length of $5.1 \pm 0.5$ characters. The length of the text was deliberately too long and the evaluation was stopped after 10 min regardless of how many words had been copied. The text was randomly allocated to ensure that each one was not always associated with the same condition. Participants were not given any advice regarding WPS usage strategy and were told to use the WPS as they wished. They were instructed to correct any errors. The same evaluation was performed again at D15 and D30.

All assessments were performed by the same investigator and were videotaped. The videos were used for the analysis.

\section{Outcome measures}

At D0, D15, and D30, the following outcome measures were evaluated:

\section{Primary outcome measure}

\section{TIS (characters per minute; cpm)}

The number of correct characters was recorded at the end of the test and this result was divided by 10 . Correct characters included letters, punctuation marks, and spaces. This measure was calculated in order to evaluate the effect of training on TIS. 


\section{Secondary outcome measures}

The secondary outcome measures (number of errors, rate of WPS, and time spent training) were noted in order to explain the TIS of each group.

\section{Number of errors and rate of word prediction use}

The number of errors and the number of words selected from the word prediction list in $10 \mathrm{~min}$ were noted from the videos. The count of errors included all errors made, including those that the participant corrected during typing. The number of errors was calculated to evaluate the effect of training on errors [15]. The rate of word prediction use was calculated in order to determine if participants actually used the software.

\section{Time spent training (SELF group)}

Participants in the SELF group were asked to record the time spent training in a log-book each day. For the REHAB group, the occupational therapist recorded the time spent training.

Complementary subjective measures (perception of speed, perception of cognitive load, and satisfaction) were evaluated in order to explain WPS effectiveness.

Perception of speed and perception of cognitive load were evaluated using a 10-point visual analogue scale (VAS) in order to explain the rate of use of the WPS. Participants evaluated their speed from 0 'very slow' to 10 'very fast'. Perception of cognitive load was similarly scored from 0 , which indicated that the task involved a 'low cognitive load' to 10 , which indicated a 'high cognitive load'.

Satisfaction with task completion was evaluated using a five-point Likert scale where zero indicated 'not satisfied' and five 'very satisfied'. This assessment was only carried out for the WPS condition.

\section{Data analysis}

A power analysis was performed a priori using $G^{*}$ Power (v3.1) software [25]. A repeated-measures Multivariate analysis of variance (MANOVA) within-between interaction was selected, with a medium effect size of 0.5 , power of 0.8 , $\alpha$ level of 0.05 , three groups, and six measurements. The total sample size necessary was calculated as 38 . We thus estimated 42 participants were necessary to account for potential drop-outs. The analysis was done by intention-to-treat.

Descriptive statistics were presented as means $( \pm$ SD) for continuous variables and frequencies for categorical variables. The data had a normal distribution (Shapiro-Wilks test) and so parametric tests were used.
The MANOVA was used to evaluate the primary outcome measure (TIS) with group (REHAB, SELF, and CONTROL) as the first factor, time (D0, D15, and D30) as the second factor and test condition (WITH and WITHOUT) as the third factor. MANOVA tests were used to evaluate the secondary outcome measures (number of errors, rate of predicted word use, satisfaction, cognitive load perception, and speed perception) with group (REHAB, SELF, and CONTROL) as the first factor, time (D0, D15, and D30) as the second factor and test condition (WITH and WITHOUT) as the third factor. A Fisher posthoc test was carried out to determine significant differences between groups, times, and conditions. The level of significance was fixed at $p<0.05$. Data were analysed using STATISTICA_10_software (StatSoft Inc. Software, Tulsa, USA).

\section{Results}

\section{Participants}

Of the 100 eligible participants who were invited to take part in the study, 42 participated (6 females and 36 males, mean \pm SD age of $39.8 \pm 12$ years). Four subsequently dropped out and 38 participants completed the study. Participants were randomised to one of the three groups after completing the initial evaluation on D0. Figure 1 shows the trial flowchart.

Participant characteristics are shown in Table 1.

\section{Time spent training}

The median (interquartile range) time spent on training for the SELF group was $5 \mathrm{~h}[0.7 ; 6]$ a month and all participants of the REHAB group spent $9 \mathrm{~h}$ a month on training.

\section{Results of the primary outcome measure}

\section{TIS}

Table 2 shows the mean TIS during the three evaluations for the three conditions.

The REHAB group typed significantly faster than the other groups with and without WPS. All three groups improved significantly with time, but the REHAB group had the largest improvement. At the end of the 30 days, the mean $(95 \%$ confidence interval (CI)) difference in increase in TIS between the CONTROL and SELF groups was 3.8 [ -1.7 to 9.4$] \mathrm{cpm}(p=0.23)$, between the REHAB group and SELF group was 12.9 [7.4 to 18.4$] \mathrm{cpm}(p<0.001)$, and between the REHAB group and CONTROL group was 9.1 [3.5 to 14.6$] \mathrm{cpm}(p<0.001)$. 
Fig. 1 Flowchart of the controlled study

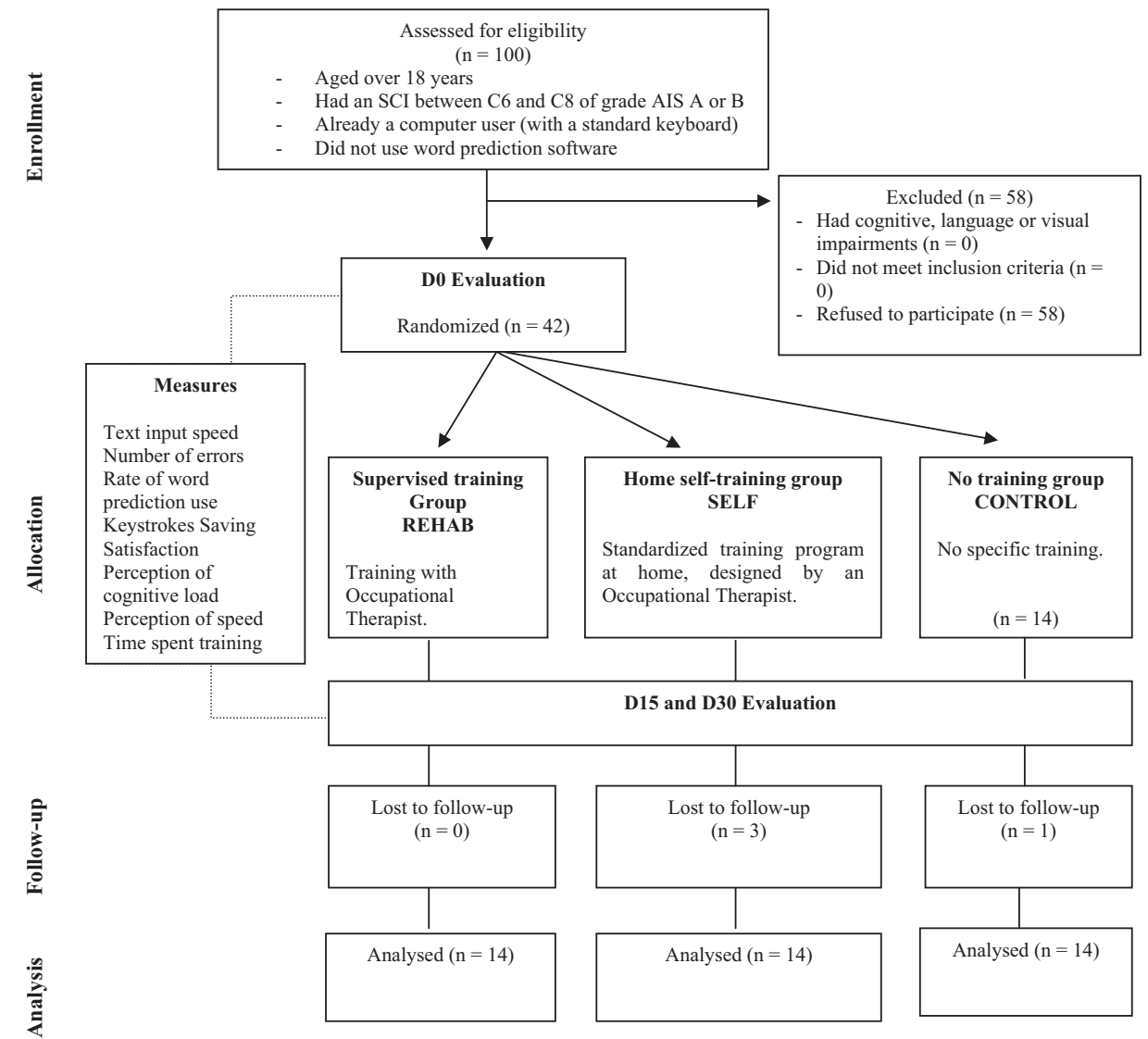

Table 1 Demographic characteristics of the participants

$\frac{\text { REHAB }}{N \text { Mean SD }} \frac{\text { SELF }}{N \text { Mean SD }} \frac{\text { CONTROL }}{N \text { Mean SD }}$

Sex

\begin{tabular}{|c|c|c|c|c|c|c|c|}
\hline Male & 12 & & & 13 & & 11 & \\
\hline Female & 2 & & & 1 & & 3 & \\
\hline Age (years) & & 38.7 & 13 & 41 & 11 & 39.6 & 12 \\
\hline $\begin{array}{l}\text { Level of education } \\
\text { (years) }\end{array}$ & & 13.1 & 2 & 13.6 & 4 & 13.8 & 3 \\
\hline $\begin{array}{l}\text { Time since lesion } \\
\text { (years) }\end{array}$ & & 12.3 & 12 & 12.3 & 10 & 5 & 4 \\
\hline \multicolumn{8}{|c|}{ Years of computer use } \\
\hline Over 10 years & 12 & & & 11 & & 10 & \\
\hline $\begin{array}{l}\text { Between } 5 \text { and } \\
10 \text { years }\end{array}$ & 1 & & & 2 & & 3 & \\
\hline $\begin{array}{l}\text { Between } 1 \text { and } 5 \\
\text { years }\end{array}$ & 1 & & & 1 & & 1 & \\
\hline \multicolumn{8}{|c|}{ Frequency of use of word processing software } \\
\hline $\begin{array}{l}>3 \text { times per } \\
\text { week }\end{array}$ & 2 & & & 4 & & 5 & \\
\hline $\begin{array}{l}\leq \text { Times per } \\
\text { week and }>\text { once } \\
\text { per week }\end{array}$ & 4 & & & 3 & & 2 & \\
\hline$<$ Once per week & 8 & & & 7 & & 7 & \\
\hline
\end{tabular}

\section{Results of the secondary outcome measures}

\section{Number of errors}

Table 3 shows the mean (95\% CI) number of errors during the three evaluations for each of the three conditions.

In all groups, participants made fewer errors with than without WPS $(p=0.004)$.

\section{Rate of WPS use}

At the end of the 30 days, the mean $(95 \% \mathrm{CI})$ difference in increase in rate of WPS use between the CONTROL and SELF groups was -6.8 [ -27.6 to 13.9$]$ ] $(p=0.70)$, between the REHAB group and SELF group was 0.8 [ -19.6 to 21] $(p=0.99)$, and between the REHAB group and CONTROL group was $7.6[-11$ to 27.2$](p=0.60)$.

\section{Complementary subjective measures}

\section{Perception of cognitive load}

At the end of the 30 days, the mean $(95 \% \mathrm{CI})$ difference in increase in perception of cognitive load between the CONTROL and SELF groups was $-0.1[-1.8$ to 1.59$]$ 
Table 2 Mean (SD) TIS during the three evaluations with and without WPS (characters/minute)

\begin{tabular}{llrlr}
\hline Time & Conditions & \multicolumn{2}{l}{ Mean (SD) TIS } & \\
\cline { 3 - 5 } & & CONTROL & SELF & REHAB \\
\hline Day 0 & Without WPS & $56.3(30)$ & $61.3(26)$ & $60.9(21)$ \\
Day 15 & & $57.3(31)$ & $61.6(25)$ & $66.3(26)$ \\
Day 30 & & $61(33)$ & $61.8(26)$ & $73.4(27)$ \\
Day 0 & With WPS & $46(19)$ & $50.1(19)$ & $51.4(17)$ \\
Day 15 & & $49.3(19)$ & $50.8(19)$ & $59(18)$ \\
Day 30 & & $61(32)$ & $52.3(19)$ & $67.4(22)$ \\
\hline
\end{tabular}

WPS word prediction software, TIS text input speed

Table 3 Mean (SD) number of errors during the three evaluations with and without WPS

\begin{tabular}{llrrr}
\hline Time & Conditions & \multicolumn{3}{c}{ Mean (SD) number of errors } \\
\cline { 3 - 5 } & & CONTROL & SELF & REHAB \\
\hline Day 0 & Without WPS & $14(8)$ & $15.2(8)$ & $17(12)$ \\
Day 15 & & $14.2(8)$ & $12.3(5)$ & $15.5(8)$ \\
Day 30 & & $15.2(8)$ & $12.4(8)$ & $15(10)$ \\
Day 0 & With WPS & $10(6)$ & $14.6(7)$ & $16.4(7)$ \\
Day 15 & & $9.8(5)$ & $10.3(6)$ & $11(8)$ \\
Day 30 & & $11.8(5)$ & $11(6)$ & $10.5(5)$ \\
\hline
\end{tabular}

WPS word prediction software

$(p=0.98)$, between the REHAB group and SELF group was $-0.61[-2.3$ to 1.07] $(p=0.64)$, and between the REHAB group and CONTROL group was $-0.48[-2.1$ to $1.1](p=0.74)$.

\section{Perception of speed}

At the end of the 30 days, the mean (95\% CI) difference in increase in perception of speed between the CONTROL and SELF groups was 0.04 [ -2.18 to 2.26$](p=0.99)$, between the REHAB group and SELF group was $0.95[-1.22$ to 3.14] $(p=0.53)$, and between the REHAB group and CONTROL group was $0.9[-1.16$ to 3$](p=0.53)$.

\section{Satisfaction}

In the REHAB group, satisfaction improved significantly with time and satisfaction was higher than in the CONTROL $(p=0.02)$ and SELF groups $(p=0.03)$.

\section{Discussion}

The aim of this study was to compare two different training protocols and a control group: a supervised training protocol, a self-training protocol and a control group who received no training. We hypothesised that the supervised protocol would lead to the greatest improvements in TIS based on data in the literature [14, 18, 19].

The results supported our hypotheses: (a) the training protocols improved TIS in participants compared with no training protocol and (b) the training protocol supervised by the occupational therapist increased TIS more than the selfrehabilitation home protocol designed (but not supervised) by an occupational therapist.

\section{Effect of training}

There was a significant effect of supervised training on TIS, which increased over time (Table 2). This was associated with an increase in the rate of WPS use, the perception of an increase in TIS, and participant satisfaction. The perception of cognitive load and the number of errors also decreased over time. These results are consistent with other studies in the literature $[14,19,20,26]$, although they contrast with Laffont et al. [21] who found mixed effects of training on TIS in 10 people with sensorimotor impairments. These differences could be attributed to the fact that the sample included in that study was more heterogeneous than the sample in the present study, and also the evaluation was carried out after 2 months of self-rehabilitation, rather than at the end of the supervised training period, as in the present study. Supervision of training seems to increase motivation and the effectiveness of training. However, the REHAB group also spent 55\% more time training on the computer: the increase in TIS is likely due to this combination of the time spent training and the supervision.

The home self-training protocol had no effect on TIS. This could be attributed to the lower intensity of training in the SELF than the REHAB group. However, the intensity of the home self-training protocol was based on an unpublished survey of the maximum training intensity that people with tetraplegia considered reasonable for self-directed work at home. Three of the four people who dropped out of the study were in the SELF group, which seems to support the idea that training for $>15$ min per day would probably be excessive for a home-training protocol without professional support. This is in accordance with previous work that showed large differences in the capacity for selftraining in individuals with SCI using WPS [22]. Therefore, although it appears unrealistic to attempt to increase selftraining time, the addition of supervision to a home protocol may increase motivation and adherence and thus effectiveness. This could be provided by weekly teleconsultation, for example.

To our knowledge, there are no data in the literature regarding self-training in the use of assistive technologies such as WPS. Unsupervised home rehabilitation protocols 
in other domains used different training intensities and also reported varying levels of adhesion to the protocols [2729]. For future work, it may be interesting to compare the effects of different methods of home self-training (modalities and durations/intensities) that are associated (or not) with the use of the WPS on TIS.

In conclusion, the results of this study showed that a supervised training protocol increased TIS. This difference was measurable and clinically meaningful. Indeed, in a previous study, we showed that people perceived that they were typing faster with a difference of 10 characters between two text entry conditions [15]. Also, this difference showed that training with an occupational therapist was important. In the light of these results, we recommend that training be provided by occupational therapists when people with tetraplegia are prescribed systems such as WPS to increase TIS.

\section{Effect of WPS}

WPS only increased TIS in the supervised training group (REHAB). This may be due to the fact that WPS was associated with a higher perception of cognitive load and a lack of perception of increased speed. Nevertheless, in all groups, the number of errors was lower with WPS than without. This is in line with the results of a previous study by our group [30]. Individuals with tetraplegia and their health-related professionals should determine the benefits of WPS relative to the time spent training.

In comparison with other supervised training protocols proposed in the literature for individuals with tetraplegia or other sensorimotor impairments [14, 18, 21], the present training protocol had the longest duration and the largest number of sessions (1 month, with three 45-min sessions per week). The results obtained were not different from those reported in the literature. Therefore, it is possible that to become skilled in the use of WPS, so that it has an impact on TIS, requires a longer training time than that proposed.

\section{Limits}

The difference between the REHAB and SELF groups may, in part, be due to differences in the intensities of training rather than the type of training per se. The use of different computers for the training and evaluation might also influence the outcomes. Nevertheless, since improvements were found in the REHAB group, the influence of the computer on participant performance seems small.

The results of this study showed that occupational therapist-supervised training improved TIS with and without WPS. These results indicate that training protocols supervised by an occupational therapist should be provided to participants with $\mathrm{C} 6-\mathrm{C} 8$ tetraplegia.

\section{Data archiving}

The datasets generated and analysed during the current study are available from the corresponding author on reasonable request.

Acknowledgements We sincerely thank all the participants in this study and the Paul Bennetot Fondation (Matmut Group Foundation).

Author contributions SP was responsible for designing the protocol, writing the protocol and manuscript, conducting the research, extracting and analysing data, interpreting results, and writing the manuscript. DB, NR, IV, AG, and SP wrote the protocol, reported and analysed the data, interpreted results, and wrote the manuscript.

\section{Compliance with ethical standards}

Conflict of interest The authors declare that they have no conflict of interest.

Statement of ethics The study was approved by the local ethics committee (CPP Ile de France XI, no. 12053).

Publisher's note: Springer Nature remains neutral with regard to jurisdictional claims in published maps and institutional affiliations.

\section{References}

1. Albert T, Ravaud J. Original article Rehabilitation of spinal cord injury in France: a nationwide multicentre study of incidence and regional disparities. Spinal Cord. 2005;43:357-65.

2. Jackson AB, Dijkers M, Devivo MJ, Poczatek RB. A demographic profile of new traumatic spinal cord injuries: change and stability over 30 years. Arch Phys Med Rehabil. 2004;85:1740-8.

3. Houlihan BV, Drainoni M-L, Warner G, Nesathurai S, Wierbicky J, Williams S. The impact of internet access for people with spinal cord injuries: a descriptive analysis of a pilot study. Disabil Rehabil. 2003;25:422-31.

4. Mattar AAG, Hitzig SL, McGillivray CF. A qualitative study on the use of personal information technology by persons with spinal cord injury. Disabil Rehabil. 2015;37:1362-71.

5. Betke M, Gips J, Fleming P. The camera mouse: visual tracking of body features to provide computer access for people with severe disabilities. IEEE Trans Neural Syst Rehabil Eng. 2002;10:1-10.

6. Folan A, Barclay L, Cooper C, Robinson M. Exploring the experience of clients with tetraplegia utilizing assistive technology for computer access. Disabil Rehabil Assist Technol. 2013; 10:1-7.

7. Kim D-G, Lee B-S, Lim S-E, Kim D-A, Hwang S-I, Yim Y-L, et al. The selection of the appropriate computer interface device for patients with high cervical cord injury. Ann Rehabil Med. 2013;37:443-8.

8. Simpson T, Gauthier M, Prochazka A. Evaluation of tooth-click triggering and speech recognition in assistive technology for computer access. Neurorehabil Neural Repair. 2010;24:188-94.

9. Koester HH, Arthanat S. Text entry rate of access interfaces used by people with physical disabilities: a systematic review. Assist. Technol. 2018;30:151-63. https://doi.org/10.1080/10400435.2017. 1291544.

10. Pouplin S, Roche N, Vaugier I, Cabanilles S, Hugeron C, Bensmail D. Text input speed in persons with cervical spinal cord injury. Spinal Cord. 2016;54:158-62. 
11. Alcantud F, Dolz I, Gaya C, Martín M. The voice recognition system as a way of accessing the computer for people with physical standards as usual. Technol Disabil. 2006;18:89-97.

12. Pouplin S, Roche N, Hugeron C, Vaugier I, Bensmail D. Recommendations and settings of word prediction software by health-related professionals for patients with spinal cord injury: a prospective observational study. Eur J Phys Rehabil Med. 2016;52:48-56.

13. Ville I, Ravaud JF. Subjective well-being and severe motor impairments: the Tetrafigap survey on the long-term outcome of tetraplegic spinal cord injured persons. Soc Sci Med. 2001; 52:369-84.

14. Koester HH, Levine SP. Effect of a word prediction feature on user performance. Augment Altern Commun. 1996;12:155-68.

15. Pouplin S, Roche N, Antoine J-Y, Vaugier I, Pottier S, Figere M, et al. The effect of word prediction settings (frequency of use) on text input speed in persons with cervical spinal cord injury: a prospective study. Disability and Rehabilitation. 2016;39: 1215-20. https://doi.org/10.1080/09638288.2016.1193229.

16. Pouplin S, Roche N, Vaugier I, Jacob A, Figere M, Pottier S, et al. Influence of the number of predicted words on text input speed in participants with cervical spinal cord injury. Arch Phys Med Rehabil. 2016;97:259-65.

17. Pouplin S, Roche N, Hugeron C, Vaugier I, Bensmail D. Recommendations and settings of word prediction software by health-related professionals for patients with spinal cord injury: a prospective observational study. Eur J Phys Rehabil Med. 2016;52:48-56.

18. Koester HH, Levine SP. Quantitative indicators of cognitive load during use of word prediction system. ProcRESNA Annual Conference. June, 1994, Nashville, USA.

19. MacKenzie IS, Zhang SX. The design and evaluation of a highperformance soft keyboard. Proc. SIGCHI Conf. Hum. factors Comput. Syst. CHI is limit - CHI' 99 1999;25-31.

20. Ward DJ, Blackwell AF, MacKayDJC. Dasher - a data entry interface using continuous gestures and language models. Proc. 13th Annu. ACM Symp. User interface Softw. Technol. - UIST '00 2000; 129-137.

21. Laffont I, Dumas C, Pozzi D, Ruquet M, Tissier A-C, Lofaso F, et al. Home trials of a speech synthesizer in severe dysarthria: patterns of use, satisfaction and utility of word prediction. J Rehabil Med. 2007;39:399-404.

22. Pouplin S, Robertson J, Antoine J-Y, Blanchet A, Kahloun J-L, Volle P, et al. Effect of dynamic keyboard and word-prediction systems on text input speed in persons with functional tetraplegia. J Rehabil Res Dev. 2014;51:467-79.

23. Koester HH, Levine S. Keystroke-level models for user performance with word prediction. Augment Altern Commun. 1997;13:239-57.

24. Fraval Lye M, Boutard C. Textzados. Isbergues: Ortho Edit; 2004.

25. Faul F, Erdfelder E, Lang A-G, Buchner A. G*Power: a flexible statistical power analysis program for the social, behavioral, and biomedical sciences. Behav Res Methods. 2007;39:175-91.

26. Handley-More D, Deitz J, Billingsley FF, Coggins TE. Facilitating written work using computer word processing and word prediction. Am J Occup Ther. 2003;57:139-51.

27. Harris JE, Eng JJ, Miller WC, Dawson AS. A self-administered graded repetitive arm supplementary program (GRASP) improves arm function during inpatient stroke rehabilitation: a multi-site randomized controlled trial. Stroke. 2009;40:2123-8.

28. Chumbler NR, Li X, Quigley P, Morey MC, Rose D, Griffiths P, et al. A randomized controlled trial on stroke rehabilitation: the effects on falls self-efficacy and satisfaction with care. J Telemed Telecare. 2015;21:139-43.

29. Van Straaten M, Cloud BA, Morrow MM, Ludewig PM, Zhao KD. Effectiveness of home exercise on pain, function, and strength of manual wheelchair users with spinal cord injury: a high-dose shoulder program with telerehabilitation. Arch Phys Med Rehabil. 2014;95:1810-7.

30. Pouplin S, Roche N, Antoine J-Y, Vaugier I, Pottier S, Figere M, et al. The effect of word prediction settings (frequency of use) on text input speed in persons with cervical spinal cord injury: a prospective study. Disabil Rehabil. 2016;39:1215-20. 